Editorials

\title{
Existence and Non-Existence of Super Mean Labeling on Star Graphs
}

\author{
${ }^{1}$ G. Uma Maheswari, ${ }^{2 *}$ Suzan Jabbar Obaiys, \\ ${ }^{3}$ G. Margaret Joan Jebarani, ${ }^{4}$ V. Balaji and ${ }^{5}$ Haider Raad \\ ${ }^{I}$ Dhanalakshmi Srinivasan College of Engineering and Technology, Chennai-603104, India \\ ${ }^{2}$ School of Mathematical and Computer Science, Heriot -Watt University, 62200, Malaysia \\ ${ }^{3}$ Department of Mathematics, Former Head, Auxilium College, Vellore - 632006, India \\ ${ }^{4}$ Department of Mathematics, Sacred Heart College, Tirupattur - 635 601, India \\ ${ }^{5}$ Department of Physics, Xavier University, Cincinnati, Ohio, USA
}

\author{
Article history \\ Received: 29-11-2019 \\ Revised: 09-11-2020 \\ Accepted: 12-11-2020 \\ Corresponding Author: \\ Suzan Jabbar Obaiys \\ School of Mathematical and \\ Computer Science, Heriot -Watt \\ University, 62200, Malaysia \\ Email: suzan_ye@yahoo.com
}

\begin{abstract}
In this article, we work on few open theorems on various aspects of Graph labeling. Herein, we prove that any single star graph is a super mean labeling and it is not super mean labeling for $n>4$. Special cases delivered in the sequel.
\end{abstract}

Keywords: Super Mean Labeling, Super Mean Graph, Wedge, Star

\section{Introduction}

Graph theory is one of the mathematical growing areas to simplify the solution of a problem in day today life. Graph theory can be used to modeling a problem that can be easier to see and find the solution for the problem. The graph consisting of nodes called as vertices connected by links called as edges. In the communication networks, the system (graph) is responsible for carrying the messages through the network and directing them along the right path.

One of the important areas in Graph theory is Graph labeling for more results on graph labeling can be found in (Gallian, 2010). The field of graph theory plays vital role in various fields. Graph labeling is an assignment of integers to the vertices or edges or both subject to certain conditions and it is used in many applications like coding theory, $\mathrm{x}$-ray crystallography, radar, astronomy, circuit design and communication network. We developed coding techniques for sharing secrecy through Graph labeling in many research article but here we developed the existence and nonexistence of one Graph labeling.

By a graph we mean a finite, simple and undirected one. The vertex set and the edge set of a graph $G$ denoted by $p$ and $q$ respectively. The disjoint union of two graphs $G_{1}$ and $G_{2}$ is the graph $G_{1} \cup G_{2}$ with and $E\left(G_{1} \cup G_{2}\right)=E\left(G_{1}\right) \cup E\left(G_{2}\right)$.

The disjoint union of two star $K_{1, m}$ and $K_{1, n}$ is denoted by $K_{1, m} \cup K_{1, n}$. The wedge of two star is obtained by an edge joining two first copy and second copy of two star for all $u_{i}$ and $v_{j}$ such that $\frac{f\left(u_{i}\right)+f\left(v_{j}\right)+1}{2}=2 m+2$. A Graph Labeling (GL) is an assignment of integers to the vertices edges, or both, subject to certain conditions. Labeled graphs serve as useful models for a broad range of applications such as: Coding theory, x-ray crystallography etc. Gallian (2010) fascinated by a variety of graph labeling and their applications, the researcher made a deep study and selected a super mean graph labeling to work on.

Based on the advantages of GL, we work on some open propositions on various aspects of GL. We that any single star graph is a super mean labeling and it is not super mean labeling for $n>4$. We illustrate a class of examples and special cases in the sequel.

\section{Literature Survey}

Most graph labeling methods trace their origin to one introduced by (Rosa, 1967), of one given by Graham and Sloane in 1980. A dynamic survey of graph labelings is gathered by (Gallian, 2010). The 
concept of Mean Labeling was introduced by (Somasundaram et al., 2003). Some new families of Mean graphs are discussed in (Vaidya and Bijikumar, 2010a; 2010b). The two star graph with an edge in common is a mean graph if and only if $|m-n| \leq 4$, which was proved by (Maheswari et al., 2015). The concept of super mean labeling was introduced and studied by (Jeyanthi et al., 2010a; 2010b; Ramya et al., 2013; Jeyanthi and Ramya, 2012) Uma (Maheswari et al., 2015; 2017a-b; 2018; 2019). The super meanness property of the subdivision of the H-graph and slanting ladder was studied by (Vasuki et al., 2017) and super meanness of subdivision graph of some caterpillars and some duplicate graphs were studied by (Vasuki and Nagarajan, 2011; Vasuki et al., 2017). The concept of sub super mean labeling was introduced by (Maheswari et al., 2017a-b; 2019), the word sub is used for the set of all vertices and edges is the subset of the full set 1 to $p+q$, but for super mean labeling it is equal to the full set. On total regularity of mixed graphs with order close to the more bound is given by (James and Graham, 2019). Motivated by the works we work on two star with super mean labeling and it is existing only for three cases, two star graph exist for two cases and three star graph is not existing for all the values and hence this study.

\section{Pre Requisites}

\section{Definition 2.1: Super Mean Labeling}

Let $G$ be a $(p, q)$ graph and $f: V(G) \rightarrow\{1,2,3, \ldots, p+q\}$ be an injection. For each edge $e=u v$, let $f^{*}(e)=\frac{f(u)+f(v)}{2}$ if $f(u)+f(v)$ is even and $f^{*}(e)=\frac{f(u)+f(v)+1}{2}$ if $f(u)+f(v)$ is odd. Then $f$ is called super mean labeling if $f(V) \cup\left\{f^{*}(e): e\right.$ $\in E(G)\}=\{1,2,3, \ldots, p+q\}$. A graph that admits super mean labeling is called a super mean graph.

\section{Definition 2.2: Wedge}

An edge is joining two disconnected graphs becomes connected is called wedge.

\section{Results and Discussion}

\section{Theorem 3.1}

For $n \geq 4, K_{1, n}$ is not a super mean graph.

\section{Proof}

Let $\left\{v_{1}, v_{2}\right\}$ be the bipartition of $K_{1, \mathrm{n}}$ with $v_{1}=\{u\}$ and $v_{2}$ $=\left\{u_{1}, u_{2}, u_{3}, \ldots, u_{n}\right\}$ Suppose $K_{1, n}$ is a super mean graph.

Then there exists a function $f: V(G) \rightarrow\{1,2,3, \ldots, 2 n+1\}$ be an injection. For each edge $e=u v$.
Let:

$$
f^{*}(\mathrm{e})=\left\{\begin{array}{c}
\frac{f(u)+f(v)}{2} \text { if } f(u)+f(v) \text { is even } \\
\frac{f(u)+f(v)+1}{2} \text { if } f(u)+f(v) \text { is odd } .
\end{array}\right.
$$

Then $f(V) \cup\left\{f^{*}(e): e \in E(G)\right\}=\{1,2,3, \ldots, p+q\}$.

Let $G=K_{1, n}, p=1+n, q=n$ and $p+q=2 n+1$.

There are $(n+1)$ odd integers and $n$ even integers in the set $\{1,2,3, \ldots, 2 n+1\}$.

Even integers are $2 \leq 2 k \leq 2 n$.

Odd integers are $1 \leq(2 k+1) \leq n+1$.

By assuming $f(u)$ be an even integers, there are 3 cases to be considered, $f(u)=2, f(u)=2 k, k<n f(u)=2 n$.

Case (a)

Let $f(u)=2$, if $f\left(u_{1}\right)=1$.

Then the corresponding edge label is $f^{*}\left(u u_{1}\right)=2$.

As the vertex value and the edge value get assigned the same number.

1 cannot be a pendent vertex of $K_{1, n}$ if $f(u)=2$.

Also 1 cannot take up the edge value at all.

Therefore 1 is missed, the definition of super mean labeling fails. Therefore $G$ is not a super mean graph if $f(u)=2$.

Case (b)

Let $f(u)=2 k, k>n$.

If $f\left(u_{1}\right)=1, f\left(u_{2}\right)=2$.

Then the corresponding edge label is $f^{*}\left(u u_{1}\right)=k+1$, $f^{*}\left(u u_{2}\right)=k+1$.

As the two edge labels have the same value corresponding it is considered the pendent vertices 1 and 2. Therefore $G$ is not a super mean graph if $f(u)=2 k$.

Case (c)

Now, consider the cases when $f(u)$ is an odd integer (i.e.,) $f(u)=1, f(u)=2 k+1, k<1, f(u)=2 n+1$. Let $f(u)=$ $2 n$ If $f\left(u_{n}\right)=2 n+1$.

Then the corresponding edge label is $f^{*}\left(u u_{m}\right)=2 n+$ 1. As Similarly the pendent vertex and the edge value get assigned the same number, so $2 n+1$ cannot be an pendent vertex. Suppose $2 n+1$ is an edge value.

The only number to be considered for the pendent vertex with respect to $2 n+1$ is as the edge value is $2 n-1$. So, $f\left(u_{n-1}\right)=2 n-1$.

Then the corresponding edge label is $f^{*}\left(u u_{n-1}\right)=\frac{2 n+2 n-1}{2}=\frac{4 n-1}{2}=2 n \neq 2 n+1$.

Therefore $2 n+1$ cannot be an edge value. Therefore $2 n+1$ is missed, the definition of super mean labeling fails. Therefore $G$ is not super mean graph if $f(u)=2 n$. 
Case (d)

Now, consider the cases when $f(u)$ is an odd integer (i.e.,) $f(u)=1, f(u)=2 k+1, k<1, f(u)=2 n+1$.

Let, $f(u)=1$. If $f\left(u_{n}\right)=2 m+1 . F\left(u_{n-1}\right)=2 m$.

Then the corresponding edge label is $f^{*}\left(u u_{n}\right)=n+1, f$ $*\left(u u_{n-1}\right)=2 n+1$. As two edge values with respect to two different pendent vertices of the same. $2 n$ cannot be a pendent vertex.

Suppose $2 n$ is an edge value. The only number to be considered for the pendent vertex with respect to $2 n$ as the edge value is $2 n-1$. So, $f\left(u_{n-1}\right)=2 n-1$.

Then the corresponding edge label is $f^{*}\left(u u_{n}\right)=\frac{2 n-1+1}{2}=n \neq 2 n$.

Therefore $2 n$ cannot be an edge value. Therefore $2 n$ is missed, the definition of super mean labeling fails. Therefore $G$ is not super mean graph if $f(u)=1$.

\section{Case (e)}

Let $f(u)=2 k+1, k<n$ If $f\left(u_{n}\right)=2 n+1 . f\left(u_{n-1}\right)=2 n$ shown in Fig. 1 to 4.

Then the corresponding edge label is $f^{*}\left(u u_{n}\right)=k+n$ $+1, f^{*}\left(u u_{n-1}\right)=k+n+1$. As two edge values with respect to two different pendent vertices are the same. $2 n$ cannot be a pendent vertex.

Suppose $2 n$ is an edge value.

The only number to be considered for the pendent vertex with respect to $2 n$ as the edge value is $2 n-1$. Hence $f\left(u_{n-1}\right)=2 n-1$.

Then the corresponding edge label is $f^{*}\left(u u_{n}\right)=\frac{2 n-1+2 k+1}{2}=n+k \neq 2 n$.

Therefore $2 n$ cannot be an edge value. Therefore $2 n$ is missed, Fig. 2 where the definition of super mean labeling fails. Therefore $G$ is not super mean graph if $f$ $(u)=2 k+1$ shown in Fig. 3 .

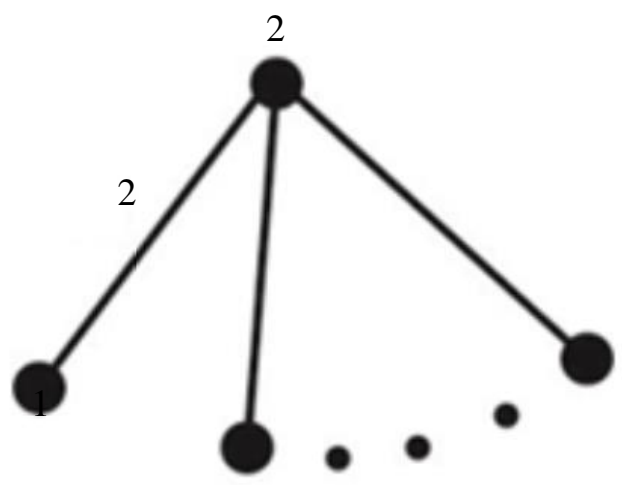

Fig. 1: $f(u)=2$

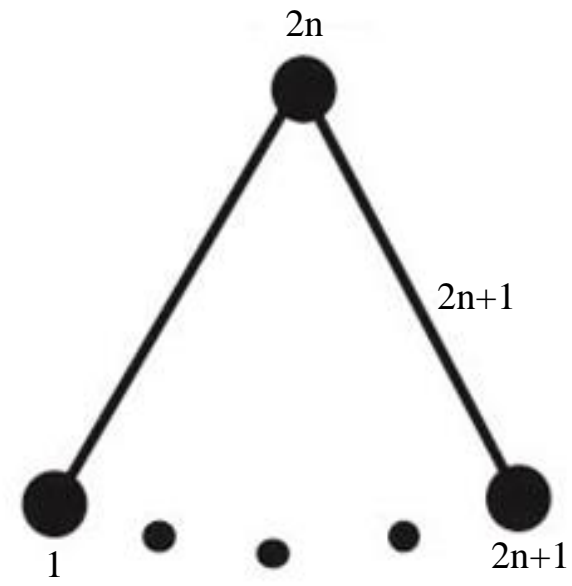

Fig. 2: $f(u)=2 n$

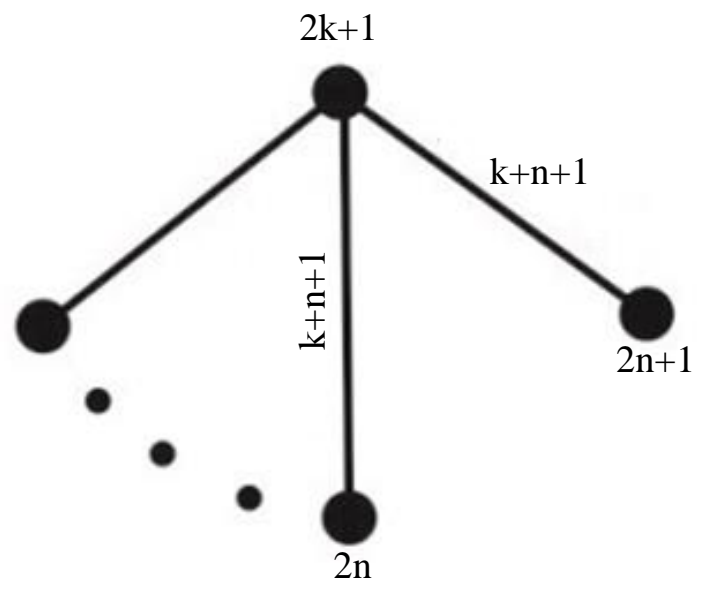

Fig. 3: $f(u)=2 k+1$

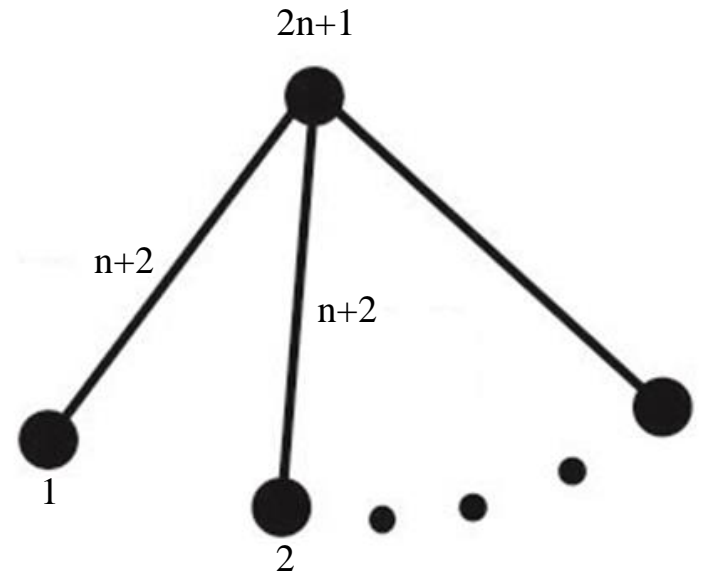

Fig. 4: $f(u)=2 n+1$. 


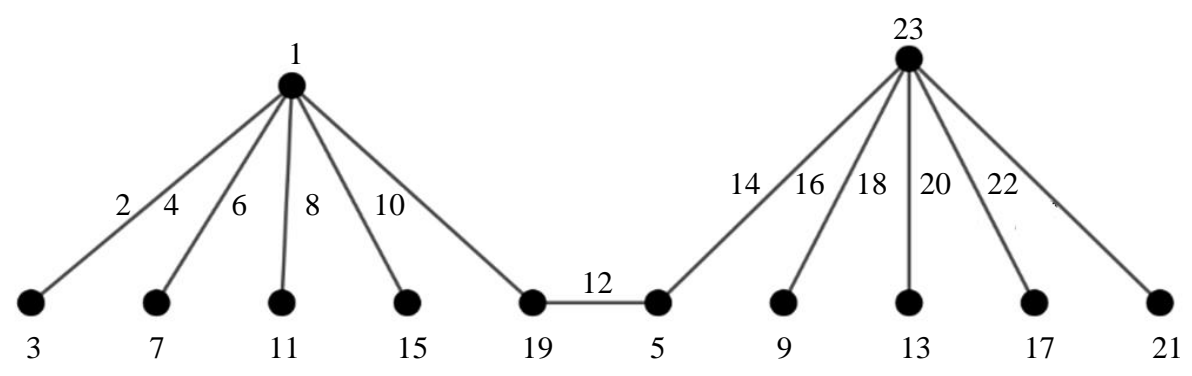

Fig. 5: $G(V, E)=K_{1,5} \cup K_{1,5}$

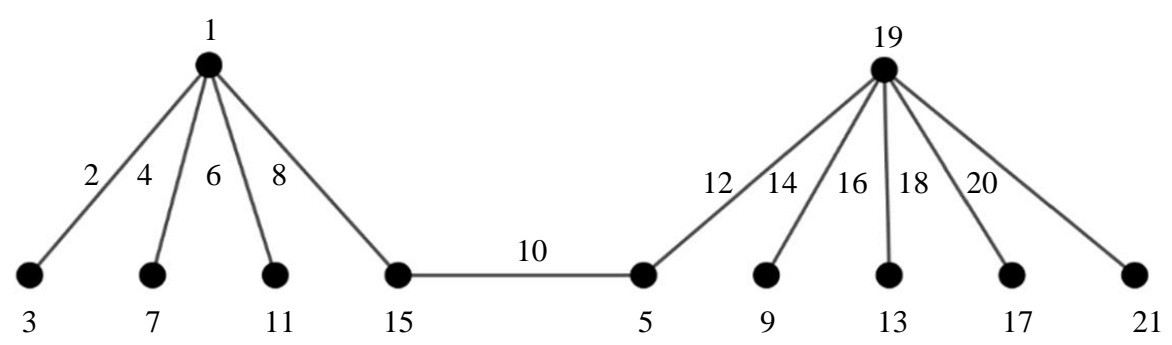

Fig. 6: $G(V, E)=K_{1,4} \cup K_{1,5}$
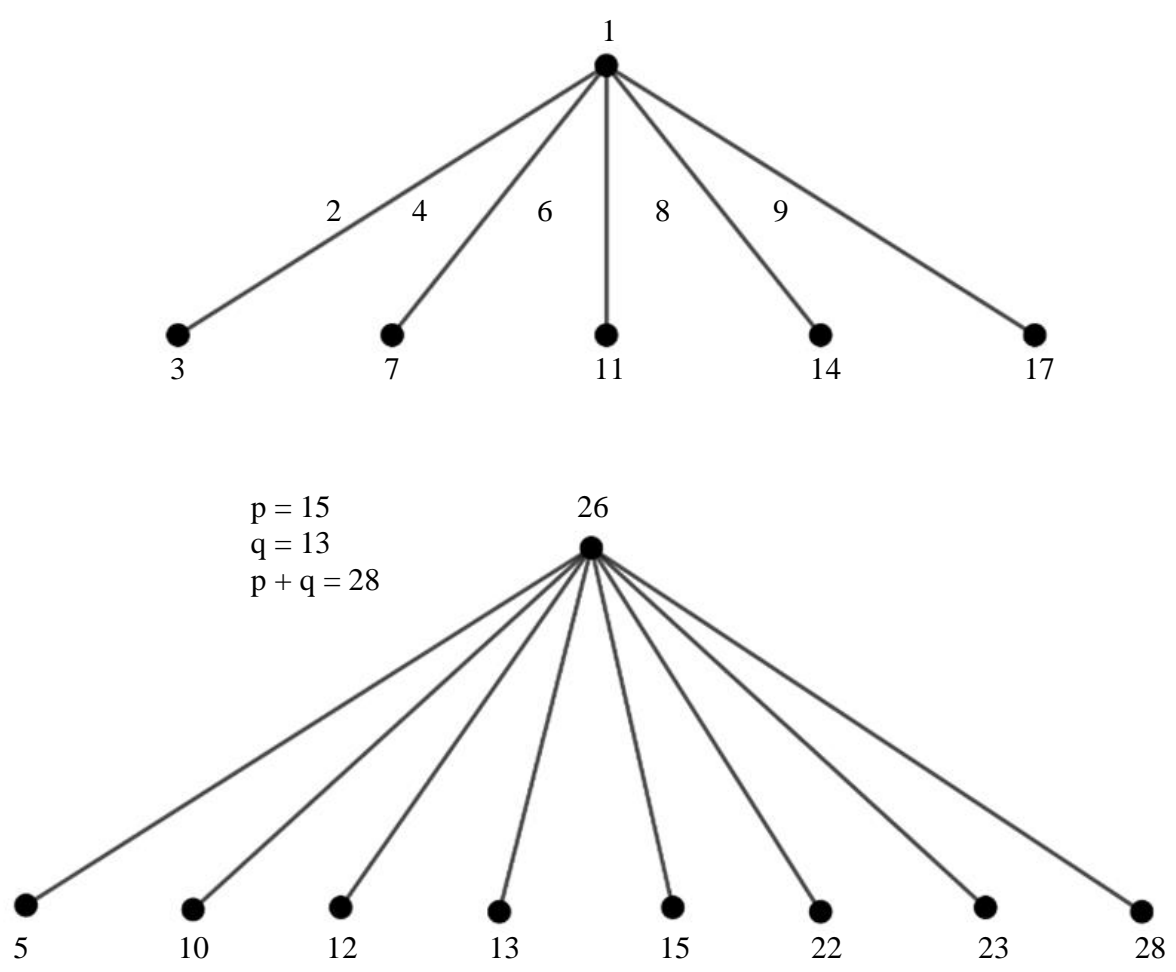

Fig. 7: Super mean labeling $K_{1,5} \cup K_{1,8}$ 

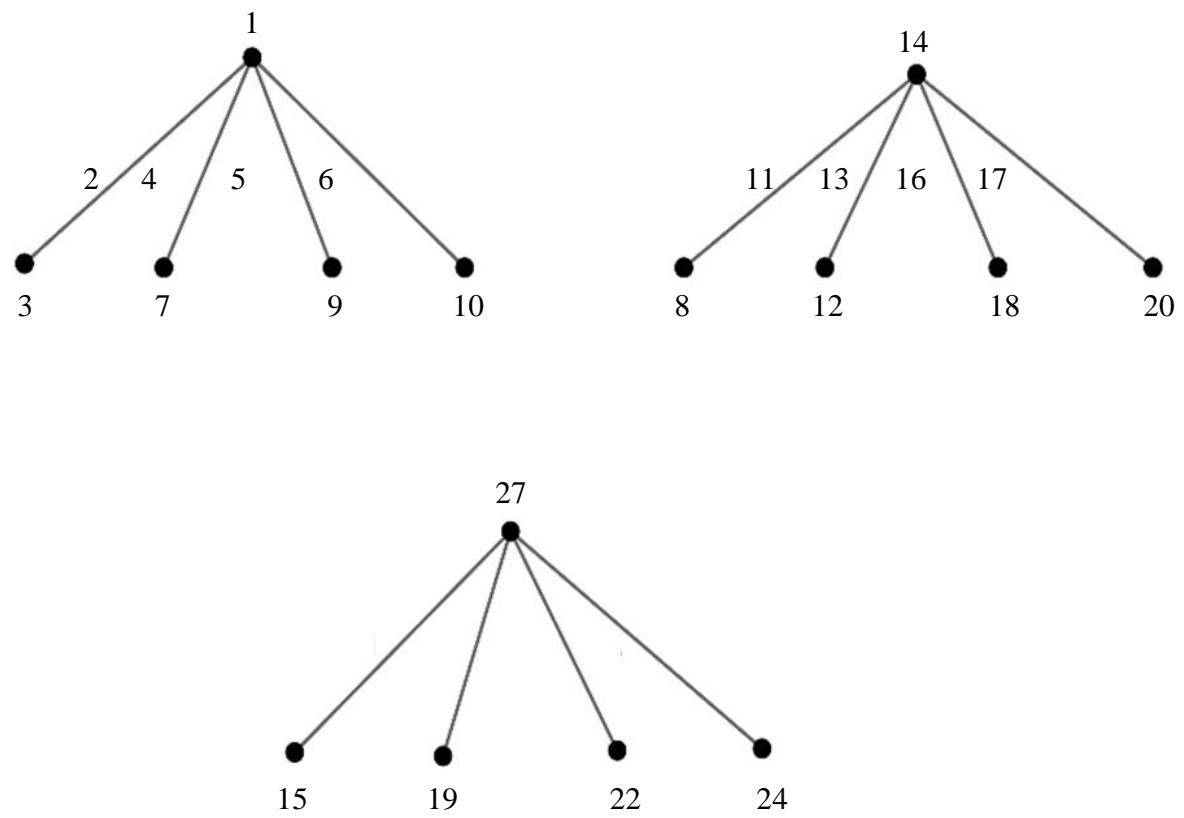

Fig. 8: Super Mean Labeling $K_{1,4} \cup K_{1,4} K_{1,4}$

\section{Case $(f)$}

Let $f(u)=2 n+1$, the largest odd integer. If $f\left(u_{1}\right)=1 . f\left(u_{2}\right)=2$ of Fig. 1 .

The corresponding edge label is $f^{*}\left(u u_{1}\right)=n+2, f^{*}$ $\left(u u_{2}\right)=n+2$. As the two edge values with respect to two different pendent vertices are the same. (1 or 2$)$ cannot be an pendent vertex also cannot assume the edge values at all. Therefore $G$ is not a super mean graph if $f(u)=2 n+1$.

The theorem concludes that the star graph $k_{1, n}$ is not a super mean graph is established by assigning all possible odd and even values of $f(u)$ For $n \leq 3$, the graph $k_{1, n}$ admits super mean labeling. We conclude that $k_{1, n}$ for $n \leq$ 4 is not a super mean graph.

\section{Theorem 3.2}

The two stars $G=K_{1, m} \cup K_{1, n}$ shown in Fig. 5-7 with an edge in common is a super mean labeling if $f|m-n| \leq 1$.

Proof: Without loss of generality, we assume that $m \leq n$.

Let us first take the case that $|m-n| \leq 1$. There are two cases viz $n=m, n=m+1$. In each case we have to prove that $G$ is a super mean labeling.

\section{Case (1)}

Let $n=m$.

Consider the graph $G=2\left(K_{1, m}\right)$ with an edge in common. Let $\{u\} \cup\left\{u_{i}: 1 \leq i \leq m\right\}$ and $\{v\} \cup\left\{v_{j}: 1 \leq j \leq m\right\}$ be the vertex set of first and second copies of $K_{1, m}$ respectively. Then $G$ has $2 m+1$ edges and $2 m+2$ vertices.

We have $V(G)=\{u, v\} \cup\left\{u_{i}: 1 \leq i \leq m\right\} \cup\left\{v_{j}: 1 \leq j \leq m\right\}$.
The required vertex labeling $f: V(G) \rightarrow\{$ $1,2,3, \ldots, 2 m+2\}$ defined as follows:

$$
\begin{aligned}
& f(u)=1 ; f(v)=4 m+3 \\
& f\left(u_{i}\right)=3+4 k, 0 \leq k \leq m-1 \text { and } 1 \leq i \leq m \\
& f\left(v_{j}\right)=5+4 k, 0 \leq k \leq m-1 \text { and } 1 \leq j \leq m
\end{aligned}
$$

The corresponding edge labeling $f^{*}: V(G) \rightarrow\{1,2$, $3, \ldots, 2 m+1\}$ defined as follows:

- The edge label of $f^{*}\left(u u_{i}\right)=2+2 k, 0 \leq k \leq m-1$ and $1 \leq$ $i \leq m$

- The edge label of $f^{*}\left(v v_{j}\right)=4+2 m+2 k, 0 \leq k \leq m-1$ and $1 \leq j \leq m$

Also the edge label of $u_{i} v_{j}$ is $2 m+2$ for all $u_{i}$ and $v_{j}$ such that $\frac{f(u)+f(v)}{2}=2 m+2$.

Therefore the edge labels of $G=\{2,4,6, . ., 2 m,+2$, $\ldots 4 m+2\}$ and has $2 m+1$ distinct edges.

Hence the induced edge labels and vertices of $G$ are distinct.

\section{Illustration (1)}

Consider the graph $G(V, E)=K_{1.5}$ with an edge in common for $m=5$.

Then: $|V|=p=12$ and $|E|=q=11$.

\section{Case (2)}

Let $n=m+1$. 
Consider the graph $G=K_{1, m} \cup K_{1, m+1}$ with an edge in common.

Let $\{u\} \cup\left\{u_{i}: 1 \leq i \leq m\right\}$ be the vertices of $K_{1, m}$ and $\{v\} \cup\left\{v_{j}: 1 \leq j \leq m+1\right\}$ be those of $K_{1, m+1}$ Then $G$ has $2 \mathrm{~m}+3$ vertices $2 m+2$ edges $+1\}$.

We have $V(G)=\{u, v\} \cup\left\{u_{i}: 1 \leq i \leq m\right\} \cup\left\{v_{j}: 1 \leq j \leq m\right.$

The required vertex labeling $f^{*}: V(G) \rightarrow\{1,2,3, \ldots, 2 m$ +3 \} defined as follows:

$$
\begin{aligned}
& f(u)=11 ; f(v)=2 m+2 n+1 \\
& f\left(u_{i}\right)=3+4 k, 1 \leq \mathrm{i} \leq m \text { and } 0 \leq k \leq m-1 \\
& f\left(v_{j}\right)=5+4 k, 0 \leq k \leq \mathrm{n}-2 \text { and } 1 \leq j \leq n-1
\end{aligned}
$$

The corresponding edge labeling $f^{*}: E(G) \rightarrow\{$ $1,2,3, \ldots, 2 m+2\}$ defined as follows:

- $\quad$ The edge label of $f^{*}\left(u u_{i}\right)=2+2 k$ for $1 \leq i \leq m$ and 0 $\leq k \leq m-1$

- $\quad$ The edge label of $f^{*}\left(v v_{j}\right)=m+n+3+2 k$ for $0 \leq k \leq$ $n-1$ and $1 \leq j \leq n$

Also the edge label of $u_{i} v_{j}$ is $2 m+2$ for all $u_{i}$ and $v_{j}$ such that $\frac{f(u)+f(v)}{2}=2 m+2$.

Therefore the edge labels of $G=\{2,4,6, \ldots, 2 m, 2 m$, $+2, \ldots, 4 m+2\}$ and has $2 m+1$ distinct edges.

In addition the edge label and vertices of $G$ are distinct. Illustration (2): Consider the graph $G(V, E)=$ $K_{1.4} \cup K_{1,5}$ with an edge in common for $m=4|V|=p=11$ and $|E|=q=10$.

Hence the graph $G$ shown in Fig. 8 is a super mean graph if $|m-n| \leq 1$

Conversely, let us take the case that $|m-n|>1$.

Suppose that $G=K_{1, m} \cup K_{1, n}$ with an edge in common for $n=m+r$ for $r \geq 2$ is a super mean graph.

Let us assume that $G=G_{1} \cup G_{2}$ with an edge in common for $G_{1}=K_{1, m}$ and $K_{1, m+r}$

Let us now consider the case that when $r=2$ and $m=1$.

Then the graph $G=K_{1,1} \cup K_{1,3}$ with an edge in common has 6 vertices and 4 edges.

Let $V(G)=\left\{v_{1, j}: 0 \leq j \leq 1\right\} \cup\left\{v_{2, j}: 0 \leq j \leq 3\right\}$ and $E(G)=$ $\left\{v_{1,0} \quad v_{1, j}=1\right\} \cup\left\{v_{2,0} v_{2, j}: 1 \leq j \leq 3\right\} \cup\left\{v_{1,1} v_{2, j}\right.$ : for any one of vertex $v_{2, j}$ for $\left.1 \leq j \leq 3\right\}$.

Suppose $G$ is a super mean graph.

Then there exists a function $f: V(G) \rightarrow\{1,2,3, \ldots, p+q\}$ be an injection. For each edge $e=u v$, let $f^{*}(e)=$ $\frac{f(u)+f(v)}{2}$ if $f(u)+f(v)$ is even and $f^{*}(e)=$ $\frac{f(u)+f(v)+1}{2}$ if $f(u)+f(v)$ is odd. Then $f$ is called super mean labeling if $f(V) \cup\left\{f^{*}(e): e \in E(G)\right\}=\{1,2,3, \ldots, p+q\}$.

Then the vertex and edge mappings of $G$ is given by $f(V) \cup\left\{f^{*}(e)=\{1,2,3, \ldots, p+q\}\right.$.
Now let us consider the following cases.

Let: $\left\{u, u_{1}\right\}$ and $\left\{v, v_{1}, v_{2}, v_{3}\right\}$ be the vertices of the graph $G=K_{1,1} \cup K_{1,3}$. We define a labeling $f: V(G) \rightarrow\{1,2$, $3, \ldots, p+q\}$ as follows:

$$
\begin{aligned}
& f(u)=1 ; \quad f(v)=7 \\
& f\left(u_{1}\right)=3 \quad f\left(v_{1}\right)=5 ; f\left(v_{2}\right)=9 ; f\left(v_{3}\right)=11
\end{aligned}
$$

Let $t_{i, j}$ be the label given to the vertex $v_{1, j}$ for $0 \leq j \leq 1$ and $V_{2, j}$ for $0 \leq j \leq 3, x_{i, j}$ be the corresponding edge label of the $V_{1,0} V_{1,1}$ and $V_{2,0} V_{2, j}$ for $1 \leq j \leq 3 \quad y_{1,1}$ be the wedge label of $t_{1,1} t_{2, j}$ for $0 \leq j \leq 3$.

\section{Case (a)}

Let us first consider $t_{1,0}=1$.

Let:

$$
\begin{aligned}
& t_{2,0}=7 \\
& t_{2,1}=5 \\
& t_{2,2}=9 \\
& t_{2,3}=11
\end{aligned}
$$

Also the wedge label of $y_{1,1}=4$ then for the corresponding edge label is $X_{1,1}=2, x_{2,1}=6, x_{2,2}=8, x_{2,3}$ $=9 t_{2,2}=9 x_{2,3}$ which is a contradiction.

It is not possible to label that two of them will induce the same label.

Therefore $G$ is a not a super mean graph. When $t_{1,0}=1$.

Case (b)

Let us next consider the case that $t_{1,0}=2$ then $t_{1,1}=4$ :

$$
\begin{aligned}
& t_{2,0}=8 \\
& t_{2,1}=6 \\
& t_{2,2}=10 \\
& t_{2,3}=11
\end{aligned}
$$

Then the corresponding edge label is $x_{1,1}=3, x_{2,1}=7$, $x_{2,2}=9, x_{2,3}=10$.

Also the wedge label of $y_{1,1}=4 t_{2,2}=10=x_{2,3}$ which is a contradiction.

It is not possible to label that two of them will induce the same label.

Therefore $G$ is a not a super mean graph. When $t_{1,0}=2$.

Case (c)

Let us next consider the case that $t_{1,0}=3$ then $t_{1,1}=5$ :

$$
\begin{aligned}
& t_{2,0}=9 \\
& t_{2,1}=7 \\
& t_{2,2}=11 \\
& t_{2,3}=1
\end{aligned}
$$


Then the corresponding edge label is $x_{1,1}=4, c_{2,1}=8$, $x_{2,2}=10, x_{2,3}=5$.

Also the wedge label of $y_{1,1}=6, t_{1,1}=5=x_{2,3}$ which is a contradiction.

It is not possible to label that two of them will induce the same label.

Therefore $G$ is a not a super mean graph. When $t_{2,0}=3$.

Case (d)

Let us next consider the case that $t_{1,0}=9$ then $t_{1,1}=6$ :

$$
\begin{aligned}
& t_{2,0}=10 \\
& t_{2,1}=8 \\
& t_{2,2}=11 \\
& t_{2,3}=1
\end{aligned}
$$

Then the corresponding edge label is $x_{1,1}=5, x_{2,1}=9$, $x_{2,2}=11, x_{2,3}=9$.

Also the edge label of $y_{1,1}=7$ :

$$
t_{2,2}=11=x_{2,2}
$$

It is not possible to label that two of them will induce the same label.

Therefore $G$ is a not a super mean graph. When $t_{1,1}=4$.

\section{Case (e)}

Let us next consider the case that $t_{1,0}=5$ then $t_{1,1}=7$ :

$$
\begin{aligned}
& t_{2,0}=11 \\
& t_{2,1}=9 \\
& t_{2,2}=2 \\
& t_{2,3}=4
\end{aligned}
$$

Then the corresponding edge label is $x_{1,1}=6, x_{2,1}=$ $10, x_{2,2}=7, x_{2,3}=8$.

Also the edge label of $y_{1,1}=8$ :

$$
t_{1,1}=7=x_{2,2} .
$$

It is not possible to label that two of them will induce the same label.

Therefore $G$ is a not a super mean graph. When $t_{1,0}=5$.

\section{Case $(f)$}

Let us next consider the case that $t_{1,0}=6$ then $t_{1,1}=8$ :

$$
\begin{aligned}
& t_{2,0}=3 \\
& t_{2,1}=10 \\
& t_{2,2}=1 \\
& t_{2,3}=5
\end{aligned}
$$

Then the corresponding edge label is $x_{1,1}=7, x_{2,1}=7$, $x_{2,2}=2, x_{2,3}=4$.

Also the wedge label of $y_{1,1}=9, x_{1,1}=7, x_{2,1}=7$. Which is a contradiction. It is not possible to label that two of them will induce the same label. Therefore $G$ is a not a super mean graph. When $t_{1,0}=60$.

\section{Case ( $g$ )}

Let us next consider the case that $t_{1,0}=7$ then $t_{1,1}=9$ :

$$
\begin{aligned}
& t_{2,0}=1 \\
& t_{2,1}=11 \\
& t_{2,2}=3 \\
& t_{2,3}=4
\end{aligned}
$$

Then the corresponding edge label is $x_{1,1}=8, x_{2,1}=6$, $x_{2,2}=2, x_{2,3}=2$.

Also the wedge label of $y_{1,1}=10, x_{2,2}=3=x_{2,3}$, which is a contradiction. It is not possible to label that two of them will induce the same label. Therefore $G$ is a not a super mean graph. When $t_{1,0}=7$.

Case (h)

Let us next consider the case that $t_{1,0}=8$ then $t_{1,1}=10$ :

$$
\begin{aligned}
& t_{2,0}=3 \\
& t_{2,1}=11 \\
& t_{2,2}=4 \\
& t_{2,3}=5
\end{aligned}
$$

Then the corresponding edge label is $x_{1,1}=9, x_{2,1}=7$, $x_{2,2}=7$.

Also the wedge label of $y_{1,1}=11, t_{2,1}=11=y_{1,1}$. Which is a contradiction. It is not possible to label that two of them will induce the same label. Therefore $G$ is a not a super mean graph. When $t_{1,0}=8$.

Case (i)

Let us next consider the case that $t_{1,0}=9$ then $t_{1,1}=11$ :

$$
\begin{aligned}
& t_{2,0}=3 \\
& t_{2,1}=1 \\
& t_{2,2}=5 \\
& t_{2,3}=7
\end{aligned}
$$

Then the corresponding edge label is $x_{1,1}=10, x_{2,1}$ $=2, x_{2,2}=4, x_{2,3}=10$. Also the wedge label of $y_{1,1}=6$, $x_{1,1}=10=x_{2,3}$. Which is a contradiction. It is not possible to label that two of them will induce the same label. Therefore $G$ is a not a super mean graph. When $t_{1,0}=9$. 


\section{Case (j)}

Let us next consider the case that $t_{1,0}=10$ then $t_{1,1}=6$ :

$$
\begin{aligned}
& t_{2,0}=5 \\
& t_{2,1}=3 \\
& t_{2,2}=7 \\
& t_{2,3}=11
\end{aligned}
$$

Then the corresponding edge label is $x_{1,1}=6, x_{2,1}=4$, $x_{2,2}=6, x_{2,3}=8$. Also the wedge label of $y_{1,1}=2$. $x_{1,1}=6$, $x_{2,2}$. Which is a contradiction. It is not possible to label that two of them will induce the same label. Therefore $G$ is a not a super mean graph. When $t_{1,0}=10$.

\section{Case (k)}

Let us next consider the case that $t_{1,0}=11$ then $t_{1,1}=1$ :

$$
\begin{aligned}
& t_{2,0}=5 \\
& t_{2,1}=3 \\
& t_{2,2}=7 \\
& t_{2,3}=10
\end{aligned}
$$

Then the corresponding edge label is $x_{1,1}=6, x_{2,1}=4$, $x_{2,2}=6, x_{2,3}=8$. Also the wedge label of $y_{1,1}=2$. $x_{1,1}=6$, $x_{2,2}$. Which is a contradiction. It is not possible to label that two of them will induce the same label. Therefore $G$ is a not a super mean graph. When $t_{1,0}=11$. $G$ is not a super mean graph all values of $t_{1,0}$. Therefore $G=K_{1,1} \cup K_{1,3}$ with an edge in common is not a super mean graph when $|m-n|=2$ Similarly, we can prove that $G=K_{1,1} \cup K_{1,4}$ with an edge in common is not a super mean graph $|m-n|=3$ Therefore, $G$ $=K_{1, m} \cup K_{1, n}$ with an edge in common is not a super mean graph if $|m-n| \geq 2$ Hence the proof.

\section{A Rule for Super Mean Labeling on Two Star Graph}

1. Some observations on super mean labeling of $K_{1, m} \cup K_{1, n}$ are listed. Here $p$ and $q$ represent the number of vertices and edges, $p=2+m+n, q=m$ $+n, p+q=2+2 m+2 n$

The numbers from 1 to $2+2 m+2 n$ must be assigned to the top vertices and the pendant vertices and in the process, the edge values get allotted. Repetition is not permitted. Here $f(u), f(v)$ and $f\left(u_{i}\right), f\left(v_{j}\right)$, are the numbers assigned to the top vertices and the pendant vertices of the first and the second star respectively. The rule for getting the edge values is $f^{*}(\mathrm{e})=\frac{f(u)+f(v)}{2}$ or $f^{*}(\mathrm{e})=\frac{f(u)+f(v)+1}{2}$ where the edge connects $u$ and $u_{i}$ : Note that the edge value can be the actual or adjusted mean.
The average of the largest and the previous number is $\frac{(2+2 m+2 n)+(2+2 m+2 n+1)}{2}=2+2 m+2 n$. As repetition is not allowed this combination is not considered. So, neither the edge value nor the pendant vertices can exceed $2+2 m+2 n$ :

2. If $\left(f(u)\right.$ and $\left.f\left(u_{i}\right)\right)$ or $\left(f(v)\right.$ and $\left.f\left(v_{j}\right)\right)$ are both odd or both even, then the edge value is the actual mean. If they are not alike then the edge value assumes the adjusted mean

3. When $f(u)$ is odd and if $f\left(u_{i}\right)=2 s f\left(u_{i+1}\right)=2 s+1$; they lead to same edge value and hence to be avoided, that is $\frac{1+6}{2}$ and $\frac{1+7}{2}$ have the same edge value 4 when $f(u)=1$

when, $f\left(u_{i}\right)=2 s+1$ and $f\left(u_{i+1}\right)=2 s+2$; they give different edge values and hence can be assigned. That is, $\frac{1+7}{2}=4$ and $\frac{1+8}{2}=5$ give different edge values. Also when $f(u)$ is even the situation is reversed. These to be noted while labeling the numbers to the pendant vertices.

Step 1: Take 1 and $p+q$ as $\mathrm{f}(\mathrm{u})$ and $f(v)$ respectively, $f\left(u_{i}\right) \neq 2$, for the edge value becomes 2 , but $f\left(v_{i}\right)$ $=2$ is permitted.

Step 2: If $f\left(u_{1}\right)=3$, then $f\left(v_{1}\right)=4$ and if $f\left(u_{1}\right)=5$, then $f\left(v_{1}\right)=2$ : That is, assign a value to $u_{1}$ and assign the next possible least integer to $v_{1}$ of the second star, the $u_{2}$ and $v_{2}$ are labeled proceeding in the same manner. Once or twice we may have to continue with assigning to $u_{i}$ 's successively in order to avoid any repetition. This procedure makes labeling a two star graph easy using super mean labeling. A two star graph with super mean labeling is given below. Hence it becomes possible to label a two star $G=K_{1, m} \cup K_{1, n}$ through super mean labeling for all values of $\mathrm{m}$ and $\mathrm{n}$ without omitting any number between 1 to $(p+q)$ but labeling is not applicable here.

\section{Theorem 3.4}

The three star graph $G=K_{1, \ell} \cup K_{1, m} \cup K_{1, n}, \ell \leq m \leq n$ is not a super mean labeling if $|m-n| \geq \ell+r, r=$ $0,1,2,3, \ldots$

\section{Proof}

We prove the theorem by the method of contradiction.

Suppose three star graph $G=K_{1, \ell} \cup K_{1, m} \cup K_{1, n}, \ell \leq m \leq$ $n$ is a super mean labeling if $m-n \mid \geq \ell+r, \Rightarrow n=\ell+m+r$.

Let us take, $r=0, \Rightarrow n=\ell+m$. 
Let us consider the graph $G=K_{1,2} \cup K_{1,2} \cup K_{1,4}$ for satisfying the condition $n=\ell+m$.

Let $\left\{u, u_{1}, u_{2}\right\}$ and $\left\{v, v_{1}, v_{2}\right\}$ and $\left\{w, w_{1}, w_{2}, w_{3}, w_{4}\right\}$ be the vertices of the graph $G=K_{1,2} \cup K_{1,2} \cup K_{1,4}$.

We define a labeling $f: V(G) \rightarrow\{1,2,3, \ldots, p+q\}$ as follows.

Let $t_{i, j}$ be the label given to the vertex $v_{1, j}$ for $0 \leq j \leq 2$ and $v_{2, j}$ for $0 \leq j \leq 3$ and $v_{3, j}$ for $0 \leq j \leq 4$ then $x_{\mathrm{i}, j}$ be the corresponding wedge label of the $v_{1,0} v_{1,1}$ for $1 \leq j \leq 2, v_{2,0}$ $v_{2, j}$ for $1 \leq j \leq 2$ and $v_{3,0} v_{3, j}$ for $1 \leq j \leq 4$.

Let $y_{1,1}$ and $y_{2,2}$ be the wedge label of first to second and second to third star graph.

Case (a)

Let us first consider $t_{1,0}=1$ :

$$
\begin{array}{lll}
t_{1,1}=3 & t_{2,0}=11 & t_{3,0}=13 \\
t_{1,2}=7 & t_{2,1}=5 & t_{3,1}=15 \\
y_{1,1}=6 & t_{2,2}=9 & t_{3,2}=19 \\
& y_{2,2}=12 & t_{3,2}=21
\end{array}
$$

The only possibility for $t_{3,4}=18$ or 20 if it is 18 then the corresponding edge values $x_{3,4}=21$ It is not possible to label that two of them will induce the same edge label. Otherwise if $t_{3,4}=20$ then the corresponding edge value is $x_{3,4}=17$ it is also not possible to label that two of them will induce same label, which is a contradiction. Similarly we can prove the graph is not super mean labeling for all the values of $t_{i, j}$ by using the proof of the theorem 2. Therefore, we conclude for least values of $r=$ 0 , similarly we can prove $r=1,2.3, \ldots$. Therefore, the three star graphs $G=K_{1, \ell} \cup K_{1, m} \cup K_{1, n}, \ell \leq m \leq n$ is not a super mean labeling if $|m-n| \geq \ell+r, r=0,1,2,3, \ldots$.

\section{Hence the proof}

\section{A Rule for Super Mean Labeling on Three Star Graphs}

Some observations on super mean labeling of $K_{1, \ell} \cup K_{1, m} \cup K_{1, n}, \ell \leq m \leq n$ are listed. Here $p$ and $q$ represent the number of vertices and edges.

$$
\begin{aligned}
& p=3+\ell+m+n, q=\ell+m+n, \\
& p+q=3+2 \ell+2 m+2 n .
\end{aligned}
$$

The numbers from 1 to $3+2 \ell+2 m+2 n$ must be assigned to the top vertices and the pendant vertices and in the process, the edge values get allotted.

Repetition is not permitted. Here $f(u), f(v), f(w), f\left(u_{i}\right)$, $f\left(v_{j}\right)$ and $f\left(w_{k}\right)$ are the numbers assigned to the top vertices and the pendant vertices and $f\left(u u_{i}\right), f\left(v v_{j}\right)$ and $f\left(w w_{k}\right)$ are the numbers assigned to the edges of the first, the second and the third star respectively.
Step 1: Take $1, \frac{p+q+1}{2}$ and $p+q$ as $f(u), f(v)$ and $f(w)$ respectively. $f\left(u_{1}\right) \neq 2$, for the edge value becomes 2 when $f\left(u_{1}\right)=2$; but $f\left(v_{1}\right)=2$ is permitted as $f(v) \neq 10$.

Step 2: If $f\left(u_{1}\right)=3$, then $f\left(v_{1}\right)=4$ and if $f\left(u_{1}\right)=5$, then $f\left(v_{1}\right)=2$ : Assigning numbers on the first star is done first then the second and third stars are done side by side. An example for super mean labeling on a three star is given below:

Allot the smallest number omitted in the first star for the first pendant vertex of the second star. Immediately, allot the next least integer to the first pendant vertex of the third star. That is $f\left(v_{1}\right)$ and $f\left(w_{1}\right)$ must be allotted one after another. This process is maintained in allotting numbers to the pendant vertices of the second star and the third star. At times one has to continue in the same star for numbering two pendant vertices, one after another. Hence it becomes possible to label a two star $K_{1, \ell} \cup K_{1, m} \cup K_{1, n}$ through super mean labeling for all values of $m$ and $n$ without omitting any number between 1 to $(p+q)$ but labeling is not applicable here.

\section{Application of Graph Labeling in Communication Networks}

The graph theory plays a vital role in various fields. One of the important area is graph labeling, used in many applications like coding theory, x-ray crystallography, radar, astronomy, circuit design, communication network addressing and data base management. Applications of labeling of graphs extend to heterogeneous fields but here we mainly focus on the communication networks. Communication network is of two types 'Wired Communication' and 'Wireless Communication'. Day by day wireless networks have been developed to easy communication between any two systems, results more efficient communication. To explore the role of labeling in expanding the utility of this channel assignment process in communication networks. In addition, graph labeling observed and identified its usage towards communication networks. We address how the concept of graph labeling can be applied to network security, network addressing, channel assignment process and social networks. Network representations play an important role in many domains of computer science, ranging from data structures and graph algorithms, to parallel and communication networks.

Geometric representation of the graph structure imposed on these data sets provides a powerful aid to visualizing and understanding the data. The graph labeling is one of the most widely used labeling methods of graphs. While the labeling of graphs perceived to be a primarily theoretical subject in the field of graph theory 
and discrete mathematics, it serves as models in a wide range of applications as listed below.

The coding theory:

- The x-ray crystallography

- The communication network addressing

- Fast communication in sensor networks using graph labeling

- Automatic channel allocation for small wireless local area network

- Graph labeling in communication relevant to adhoc networks

- Effective communication in social networks by using graphs

- $\quad$ secure communication in graphs

\section{Conclusion}

In this study we proved the super mean labeling is possible on single star graph for some values also for two star and it is not possible for all the values of three star graph. We made full advantage of it and apply graph labeling in to subfields of coding theory, cryptography. For future works, researchers may get some information related to graph labeling and its applications in communication field and work on some ideas related to their field of research.

\section{Acknowledgement}

The authors would like to thank Dr Haider Raad for his help and support

\section{Author's Contributions}

G. Uma Maheswari: Conceptualization, Methodology, writing original draft preparation and review correction.

Suzan Jabbar Obaiys, Haider Raad: Conceptualization, Methodology, Investigation, Writing original draft preparation and review correction.

G. Margaret Joan Jebarani: Investigation of result and review of the paper.

V. Balaji: Analysis, resources, review of the paper.

Haider Raad: Analysis, review and editing of the paper.

\section{Ethics}

This article is original and contains unpublished material. The corresponding author confirms that all of the other authors have read and approved the manuscript and no ethical issues involved

\section{References}

Gallian, J. A. (2010). A dynamic survey of graph labeling. The Electronic journal of combinatorics, 17, 60-62.
James, T., \& Graham E. (2019) On total regularity of mixed graphs with order close to the Moore bound. Graphs and Combinatorics, 35(6), 1253-1272.

Jeyanthi, P., \& Ramya, D. (2012). Super mean labeling of some classes of graphs. Mathematical Combinatorics, 1, 83-91.

Jeyanthi, P., Ramya, D., \& Thangavelu, P. (2010a). On super mean labeling of some graphs. SUT J. Math, 46, 53-66.

Jeyanthi, P., Ramya, D., \& Thangavelu, P. (2010b). Some constructions of k-super mean graphs. International Journal of Pure and Applied Mathematics, 56(1), 77-86.

Maheswari, G. U., Arthy, J., \& Obaiys, S. J. (2018). A Method of Secret Coding technique on Two Star Graphs. International Journal of Computer Applications, 975, 8887.

Maheswari, G. U., Jebarani, G. M. J., \& Balaji, V. (2017a). On Similar Super mean Labeling for two star GRAPH. Asian Journal of Mathematics and Computer Research, 53-62.

Maheswari, G. U., Jebarani, G. M. J., \& Balaji, V. (2017b). Coding Through a Two Star and Super Mean Labeling. In Applied Mathematics and Scientific Computing (pp. 469-478). Birkhäuser, Cham.

Maheswari, U., Obaiys, S. J., \& Jebarani, M. J. (2019). A comprehensive study of super mean labelling in three star graph.

Maheswari, V., Ramesh, D. S. T., Francis, S., \& Balaji, V. (2015). On Mean Labeling. Bulletin of Kerala Mathematics Association, 12(1), 54-64.

Ramya, D., Ponraj, R., \& Jeyanthi, P. (2013). Super mean labeling of graphs. Ars Combinatoria, 112, 65-72.

Rosa, A. (1967). On certain valuations of the vertices of a graph, Theory of Graphs (Internat. Symposium, Rome, July 1966).

Somasundaram, S., Vidhyarani, P., \& Ponraj, R. (2003). Geometric mean labeling of graphs. Bulletin of Pure $\&$ Applied Sciences-Mathematics and Statistics, 30(2), 153-160.

Vaidya, S. K., \& Bijukumar, L. (2010a). Some new families of mean graphs. Journal of Mathematics Research, 2(3), 169.

Vaidya, S. K., \& Bijukumar, L. (2010b). Mean labeling for some new families of graphs. PRAJNA-Journal of Pure and Applied Sciences, 50.

Vasuki, R., \& Nagarajan, A. (2011). Further results on super mean graphs. Journal of Discrete Mathematical Sciences and Cryptography, 14(2), 193-206.

Vasuki, R., Sugirtha, P., \& Venkateswari, J. (2017). Super mean labeling of some subdivision graphs. Kragujevac Journal of Mathematics, 41(2), 179-201. 